\title{
PRACTICAL IMPLEMENTATION FOR FUZZY SELF TUNING OF OPTIMAL PID CONTROLLER TO SERVO PERMANENT MAGNET SYNCHRONOUS MOTOR
}

\author{
M. A. Abdel Ghany ${ }^{1}$, H. Abdel Magid ${ }^{2}$, M. Abdullah Eissa ${ }^{2}$, M. E. Bahgat ${ }^{2}$ \\ and A. Bassuiny ${ }^{2}$ and Soliman Sharaf ${ }^{2}$ \\ ${ }^{1}$ Nahda University (NUB), Bani Sweif City, Egypt \\ ${ }^{2}$ Faculty of Engineering - Helwan, University of Helwan, Cairo, Egypt
}

\begin{abstract}
This paper describes the design and practical implementation for speed Fuzzy Self Tuning of Optimal PID control FSTOPID on a Servo Permanent Magnet Synchronous Motor PMSM. In this work, an industrial PMSM system has been identified including its drive. Nonlinear Least Squares Algorithm NLSA is used for model identification. For speed control, a variable load for the PMSM represents nontraditional control problem. One of the solutions to the problem is to apply a FSTOPID controller. This requires reformulating the control problem to include two parts, optimal PID controller and fuzzy logic controller FLC parts. The first part deals with the PID controller tuned using Ant Colony System ACS algorithm. The second one represents the on line fuzzy self-tuned of the optimal PID. The goal of this design is to regulate the speed and improve the transient performance of the PMSM system under load demand variations. Comparative analyses of practical implementation for the PMSM drive system are demonstrated under diverse load. Finally, experimental results show accurate identification and speed favorable performance. The results prove that the proposed controller is very useful for industrial servo PMSM system.
\end{abstract}

\section{Keywords - Permanent Magnet Synchronous Motor; Identification ; Ant Optimization Technique; PID Control Fuzzy Logic Control.}

\section{INTRODUCTION}

Permanent magnet synchronous motor PMSM has received widespread acceptance in industrial servo applications of accurate speed control, this is because of some of its outstanding features such as superpower density, high torque to current ratio, fast response and better accuracy [1-6]. PMSM are widely used in low and mid power applications such as computer peripheral equipments, robotics, adjustable speed drives and electric vehicles. The growth in the market of PMSM motor drives has demanded the need of simulation tools capable of handling motor drive simulations [7-9].

Recently many efforts have been made toward various modern control strategies for PMSM. The fuzzy control theory has succeeded in controlling complex nonlinear, incompletely modeled that are not amenable to conventional control techniques [10-12]. The PMSM fuzzy control design methods of [13-14] are based on heuristics-based fuzzy approach which is essentially model free. Even though the performances of the previous heuristics-based methods of [10-14] may be satisfactory, there are some problems. Trial and error method is usually needed to design fuzzy controllers, and stability analysis is very difficult. However, precise control of a PMSM is not easy due to nonlinearities of PMSM servo systems, 
parameter and load torque variations. Therefore, in order to enhance the performance of the PMSM drive system, identification of the PMSM drive system has been performed [15].

The PID control scheme has been used because of its simple structure, better robustness and high reliability; see for instance [16-19]. This conventional approach of such an application demands the implementation of a PID controller characterized by two main drawbacks, the slow transient response and the possible undesirable oscillations for PMSM motor drives. The optimal PID gains can reduce the effect of this problem. In addition, a combination of an optimal PID with a fuzzy logic as self tuner of the PID gains represents a hybrid controller useful for such problem for PMSM servo systems.

Based on the above, this paper provides a simple and straightforward procedure for designing FSTOPID for PMSM drive system. The main contributions of this paper are given in three steps : The first step in the design procedure is the identification of the PMSM drive system using Nonlinear Least Squares Algorithm (NLSA) [20-21]. The second step is a fine-tuning for the PID controller by using ACS optimization. The last one is a fuzzy self-tuning of the PID. The proposed controllers are applied to the PMSM. Different tests are carried out using the hardware implementation of the proposed controller. Practical experimental results show that the performance of the controller improves significantly the controlled system over severe load torque disturbance.

\section{SERVO MOTOR SYSTEM AND EXPERIMENTAL SETUP}

The experimental plat form is shown in Fig (1). The experimental setup is designed for the theoretical and practical investigation studies of the proposed PMSM controllers. The experimental setup consists of six parts:

1- SEW Synchronous 3-phase permanent magnet servo motor.

2- SEW MOVIDRIVE-B to drive the motor.

3- Mechanical load.

4- PC used to perform the control algorithms.

5- Interface adapter option USB11A.

6- A data acquisition card (DAQ) NI USB-6008.

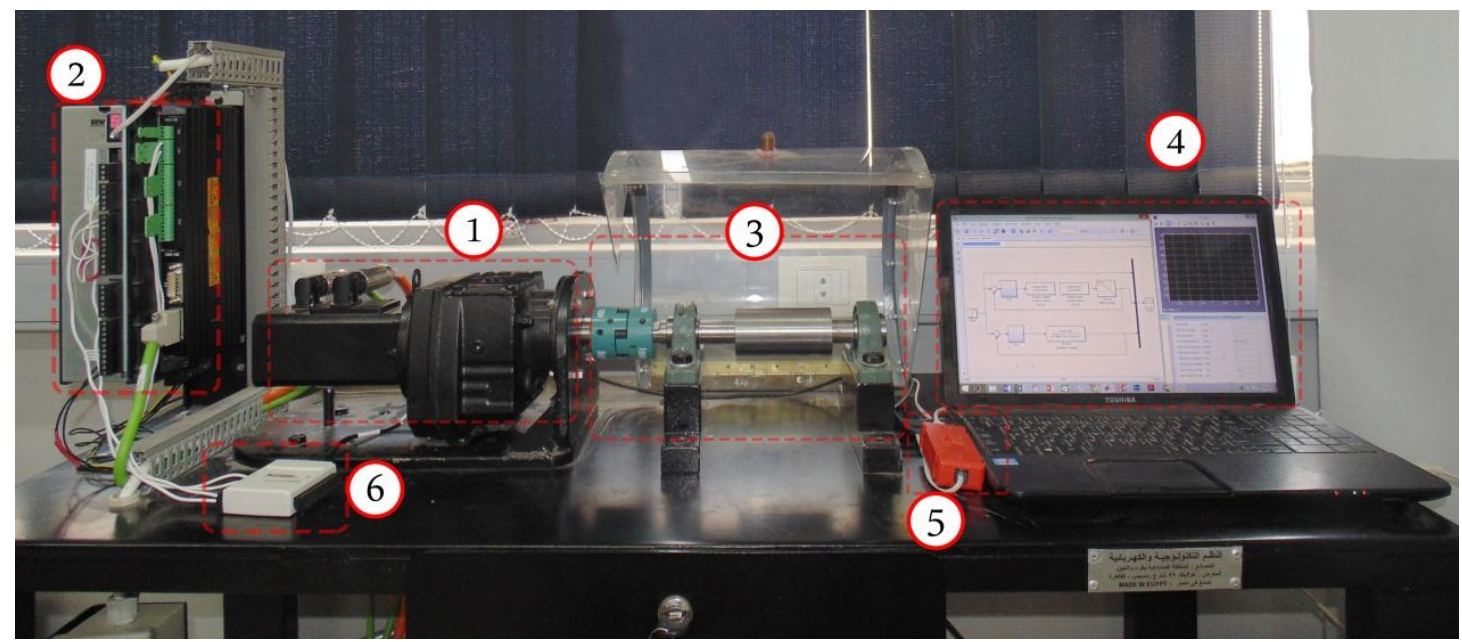

Fig (1) - Expremntal setup of servo system

The control structure of the servo drive is a fuzzy self tuning for optimal PID controller. The computer calculates the optimal PID gains and then it sends them to the servo drive at each sampling time. The applied load torque to the motor represents the external inertia load exerted on the motor. 


\section{SERVO MOTOR MODEL IDENTIFICATION}

In the expremntal setup of PMSM system, despite the availability of the motor parameters as given in appendix, some parameters of the servo motor system still unknowns as the motor drive -(The multifunctional data acquisition DAQ, shaft encoder, bearing with housing coupling) and mechanical load. Therefore, the unknown parameters of drive and mechanical load cause complexity for performing mathematical modeling for the PMSM. Based on this, system identification must be performed. An experimental modeling approach with experimental measurements data of real processes is used to determine the mathematical model. System Identification MTLAB toolbox is employed for this aim.

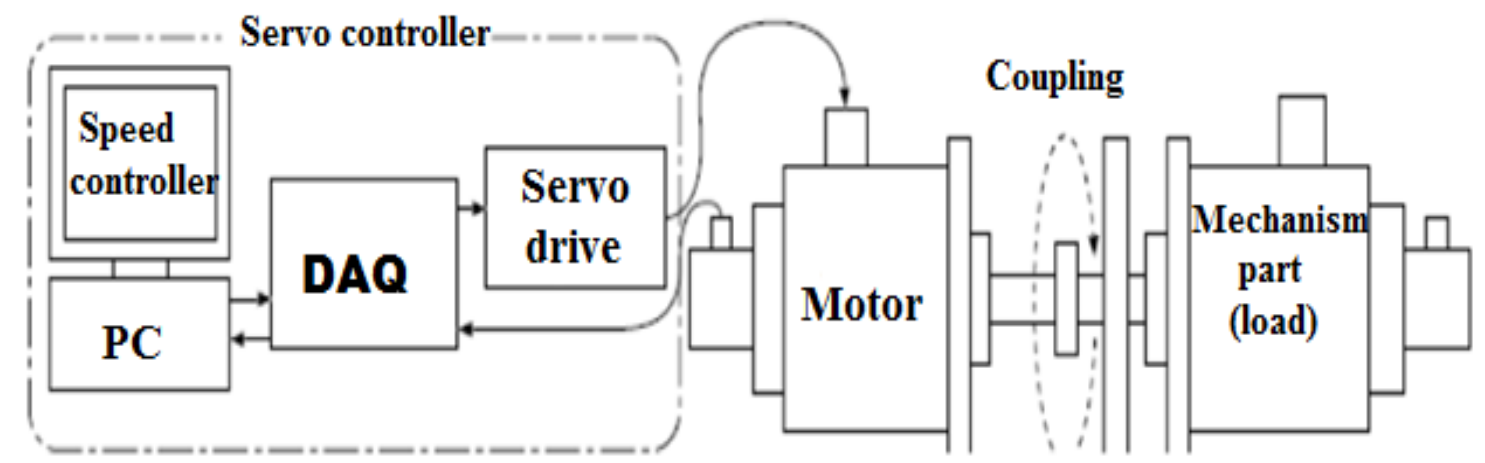

Fig (2) - Block diagram for description of servo system

\section{MODEL PARAMETERS IDENTIFICATION}

Parameter identification plays a key role in today servo drives since the identified model allows the tuning of a controller applied to a servomechanism. Among literature, there are several references dealing with parameter identification techniques [20-22]. The system identification steps can be summarized as follows :

1- Data collection

3- Validation of the obtained model
2- Estimation of the parameters

4- Choice of the model structure

\section{Data Collection}

The first step in identification process is to collect the input /output experimental data from the practical PMSM system. The purpose of this stage is to describe the system dynamics over its entire range of operation. In this work, Chirp Function is used among the system identification [20-22]. Chirp is swept-frequency cosine generator given by the following equation:

$$
x(t)=\sin \left[\phi_{o}+2 \pi f_{o}\left(\frac{k^{t}-1}{\ln (k)}\right)\right]
$$

where : $\quad \phi_{\boldsymbol{o}}$ is the initial phase (at $\left.\mathrm{t}=0\right\} \mathrm{t}=0$ ).

fo is the starting frequency (at $\mathrm{t}=0$ )

$\boldsymbol{k}$ is the rate of exponential change in frequency

The Multifunctional data acquisition (DAQ) is displayed in Fig (3). DAQ represents the terminal parts for sending and receiving identification signals. It can take a continuous signal and sampling it at evenly spaced interval to produce a series of discrete values that represent the original signal. In addition, DAQ is used to transmit the analog signal that represents the speed from the drive to the computer. 


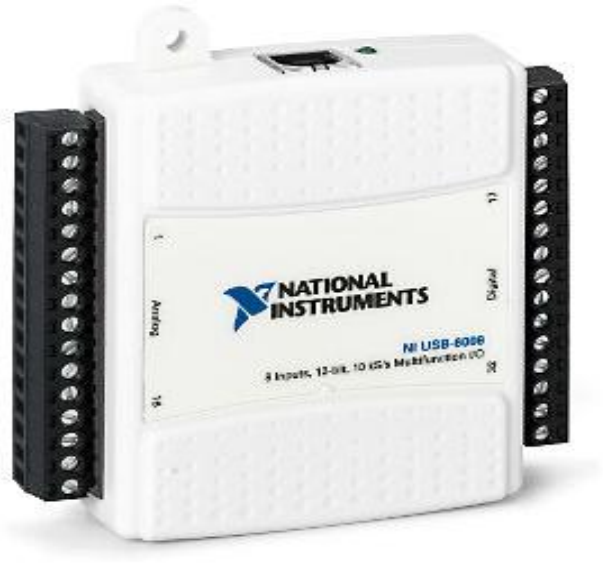

Fig (3) - Multifunctional DAQ NI USB-6008

Based on the above, the identification input signal is an input voltage $\boldsymbol{v}$ and is given by equation (3). The output signal is represented by a voltage corresponding to the measured speed $\boldsymbol{\omega}$ of motor. Figure (4) illustrates the construction of DAQ and it's simulation in the Simulink Tool box environment. The Input/output signals using Chirp Function are illustrated in Fig (5).

Real system
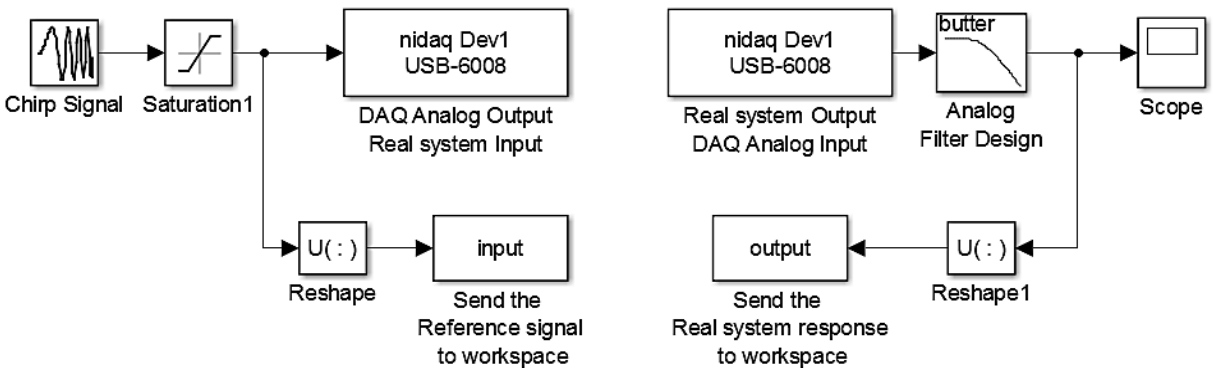

Fig (4) - Input/output collected data for the real servo system.

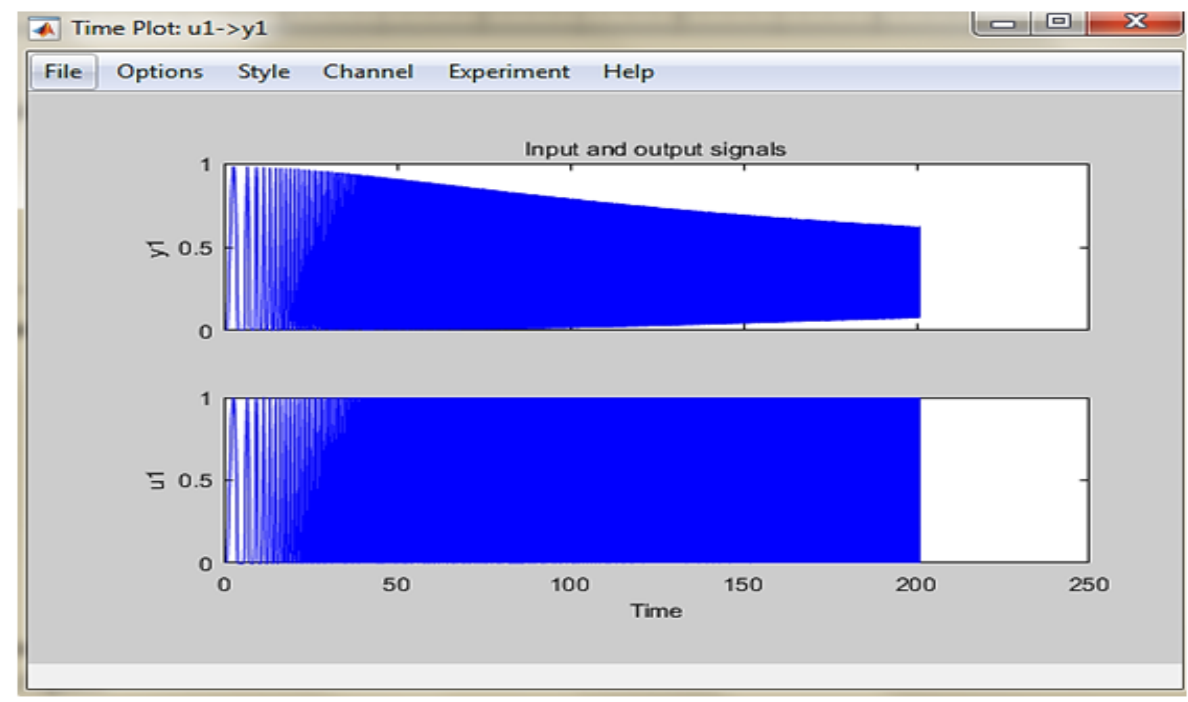

Fig (5) - Input/output collected data

Different types of PMSM identification models are performed to get the best fit model. The system identification is based on the Non-linear least square technique. The validation of the 
3-fits models (tf3, tf6, tf7) by using different types of input signals (step, square and Sin) are performed to ensure the sustainability of the model response as shown in Fig (6).

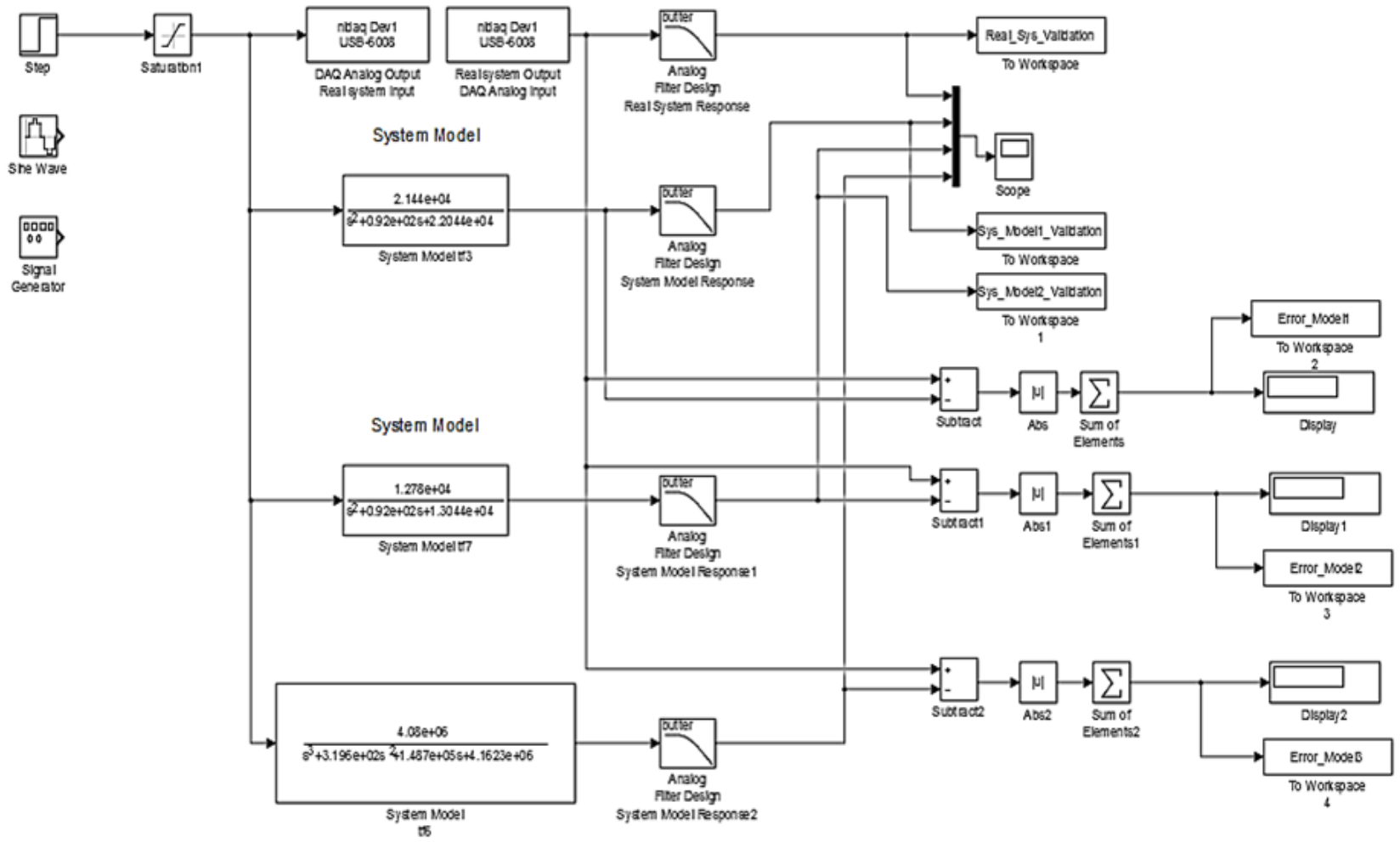

Fig (6) - Block diagram for system model validation

\section{Model Validation}

Figures (7) and (9) show the validation of the experiential output of the PMSM and the identified linear second order for models (tf3) and (tf7). In addition, figures (8) and (10) show the absolute of sum of difference between experiential outputs of the PMSM and identified linear second order for models (tf3) and (tf7).

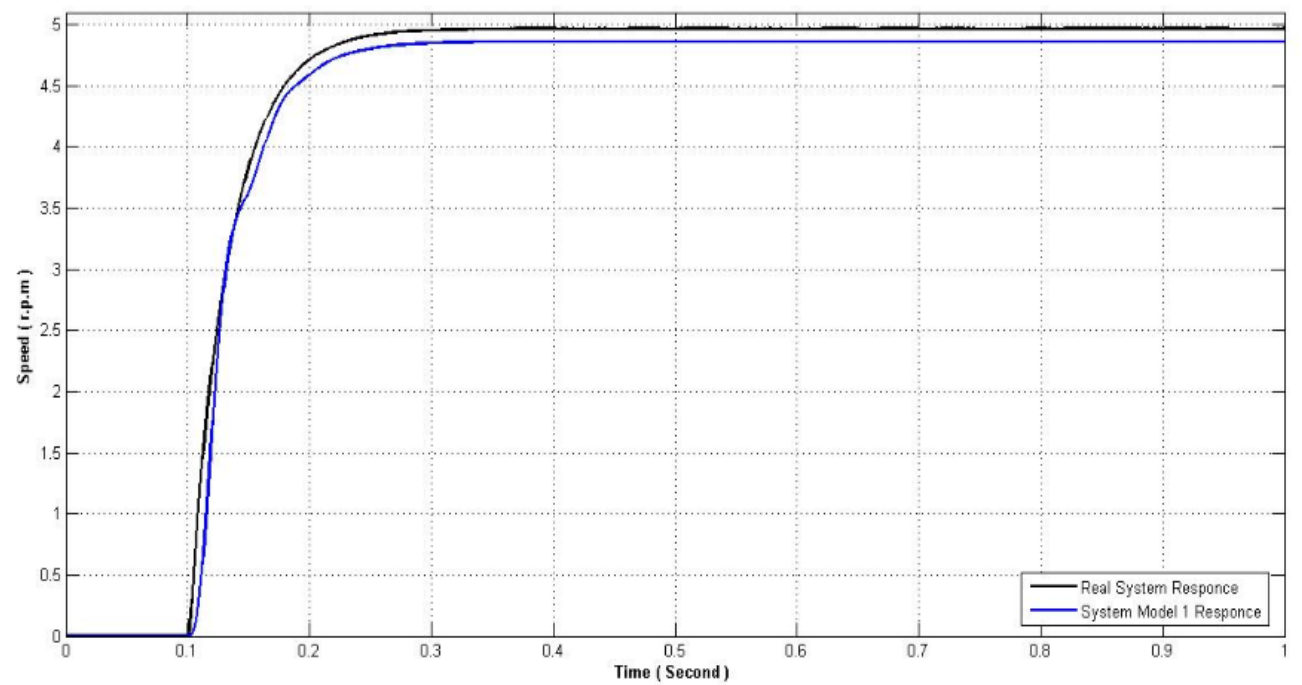

Fig (7) - System model validation for model (tf3) 


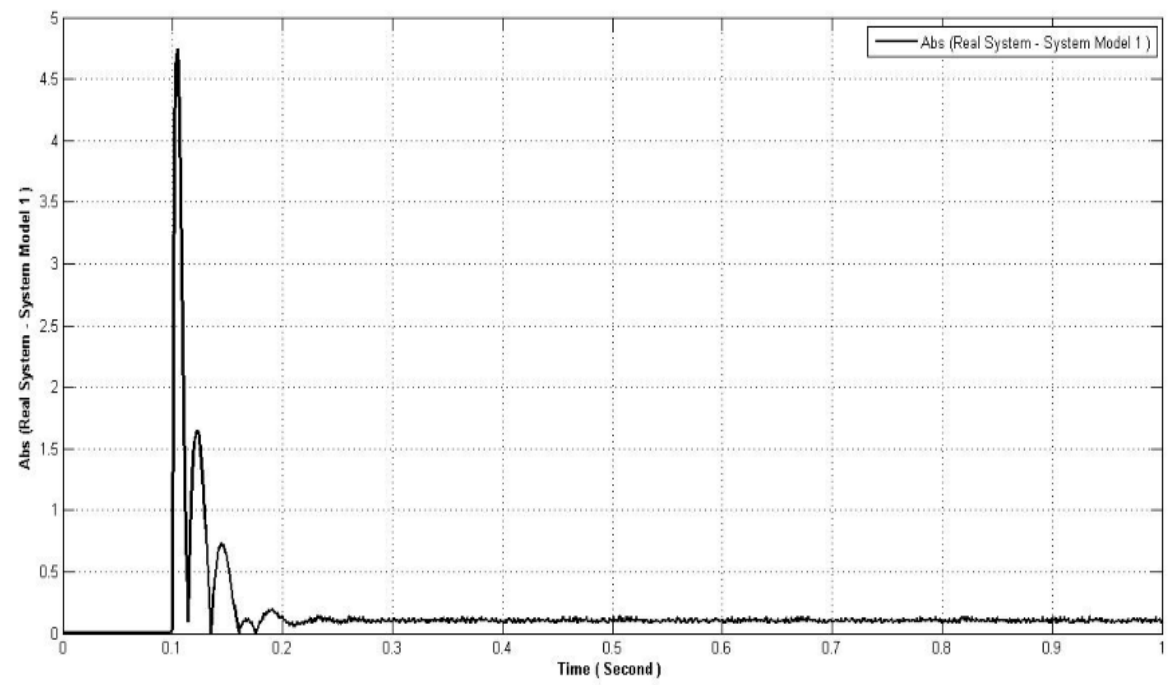

Fig (8) - The sum of the absolute value between the real and model output of PMSM for model (tf3)

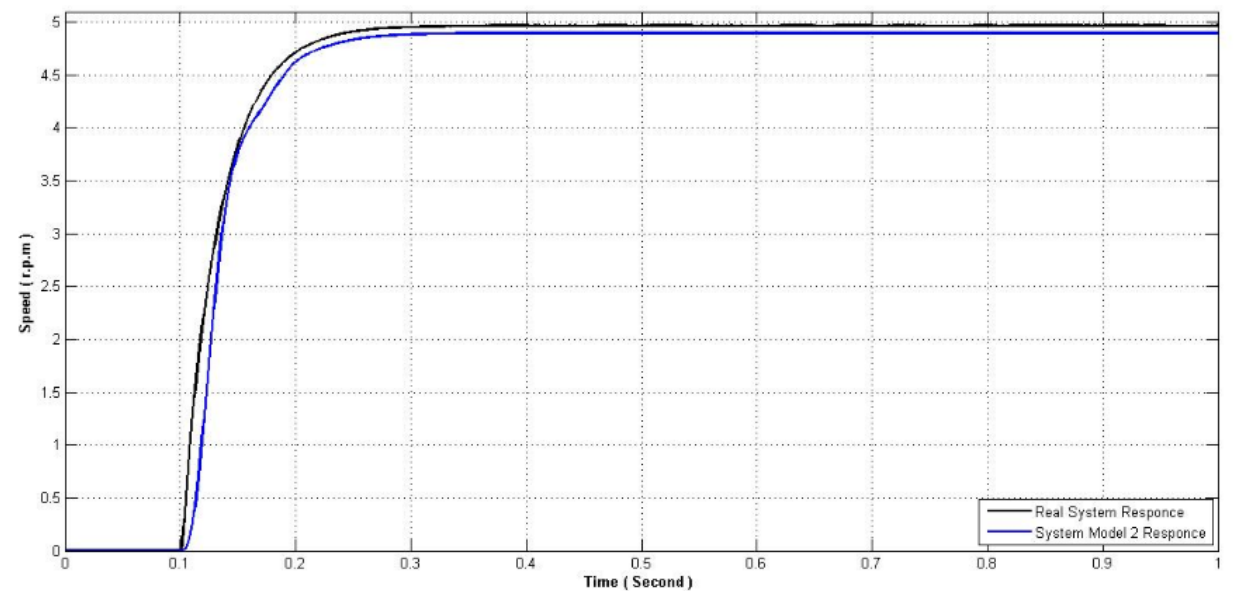

Fig (9) - System model validation for model (tf7)

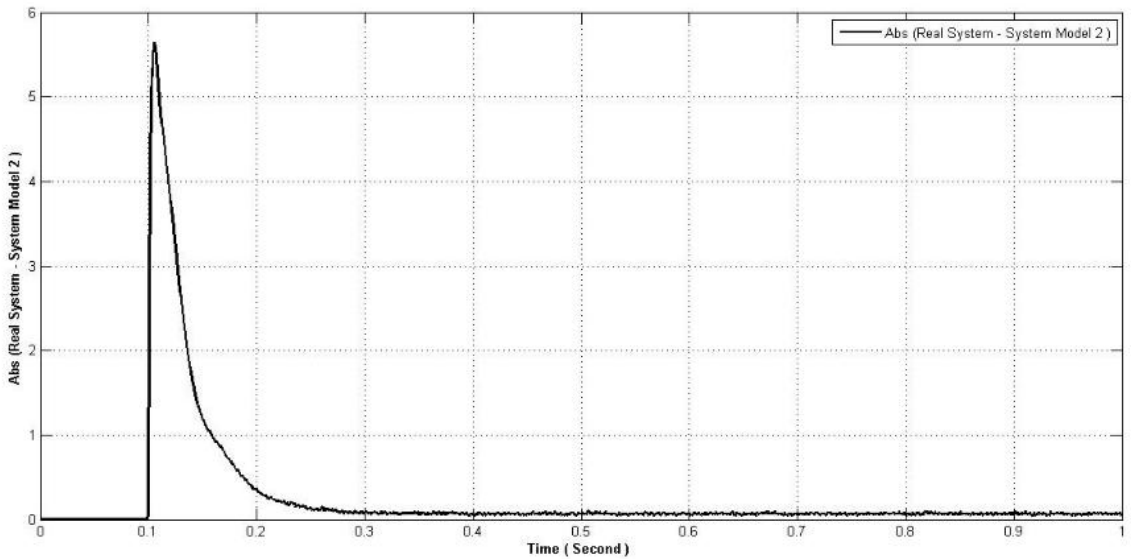

Fig (10) - The sum of the absolute value between the real and model output of PMSM for model (tf7)

\section{Model Structure}

The second order is selected as a model structure for describing the system according to wide range survey and many experimental trials (Model tf3). The approximate transfer function of the proposed servo system may be written as follows : 


$$
\frac{\omega(s)}{v(s)}=\frac{\mathbf{k}}{\mathbf{a s}+\mathbf{b s}+\mathrm{c}}
$$

where: $\quad \omega(s) \quad$ Rotor speed.

$\mathbf{v}(s) \quad$ controlled voltage.

$\mathbf{k}, \mathbf{a}, \mathbf{b}$ and $\mathbf{c}$ are the transfer function parameters.

Based on the model validation Model (tf3) is chosen and the identification parameters results of the linear models are given in Table (1).

Table (1) - The values of the parameters of the identified model.

Model The coefficients

\begin{tabular}{c|cccc}
\hline & $a$ & $b$ & $c$ & $k$ \\
TF & 1 & $.92 \mathrm{e}+02$ & $2.2044 \mathrm{e}+04$ & $2.144 \mathrm{e}+04$
\end{tabular}

The transfer function representing the angular velocity $\boldsymbol{\omega}$ of motor and the input motor voltage $\mathbf{v}$ obtained from the system identification process could be expressed as :

$$
\frac{\omega(s)}{v(s)}=\frac{2.144 \mathrm{e} 04}{\mathrm{~s}^{2}+0.92 \mathrm{e} 02 \mathrm{~s}+2.2044 \mathrm{e} 04}
$$

\section{ANT SYSTEM OPTIMIZATION AND ANT BASED PID CONTROLLER}

Ant Colony Optimization ACO that was initially proposed by Colorni , Dorigo and Maniezzo [23]. In this algorithm, computational resources are allocated to a set of artificial ants that exploit a form of indirect communication mediated by the environment to find the shortest path from the ant nest to a set target. Usually, the optimization process of automatic control consists of finding the controller parameters such that to minimize or maximize a given cost function of the closed loop system consisting of an ant based PID controller and an unknown plant. The effectiveness of the proposed ant based PID is quantified by the following performance criteria of the system $\mathrm{J} 1$ is designed as follows [24-26]:

1

$$
\mathbf{J} 1=\frac{}{\left[c_{1}\left(t_{r}-t_{r d}\right)+c_{2}\left(M_{p}-M_{p d}\right)+c_{3}\left(t_{s}-t_{s d}\right)+c_{4}\left(e_{s s}^{e_{s}}-e_{s s d}\right)\right]}
$$

Where : $\quad \mathbf{c}_{\mathbf{1}}: \mathbf{c}_{\mathbf{4}} \quad$ are positive constants (weighting factor), their values are chosen according to prioritizing their importance.

$\mathbf{t}_{\mathbf{r d}} \quad$ is the desired rise time.

$\mathbf{M}_{\mathbf{p d}} \quad$ is the desired maximum overshoot.

$\mathbf{t}_{\text {sd }} \quad$ is the desired settling time,

$\mathbf{e}_{\text {ssd }} \quad$ is the desired steady state error [24-26].

\section{Generation of nodes and ant paths}


Figure (11) depicts the closed loop control system used in this paper. Using the same technique presented in [23-26], it can be assumed that the value of PID gain parameters has five digits; one digit before decimal point and the other four digits after the decimal point.

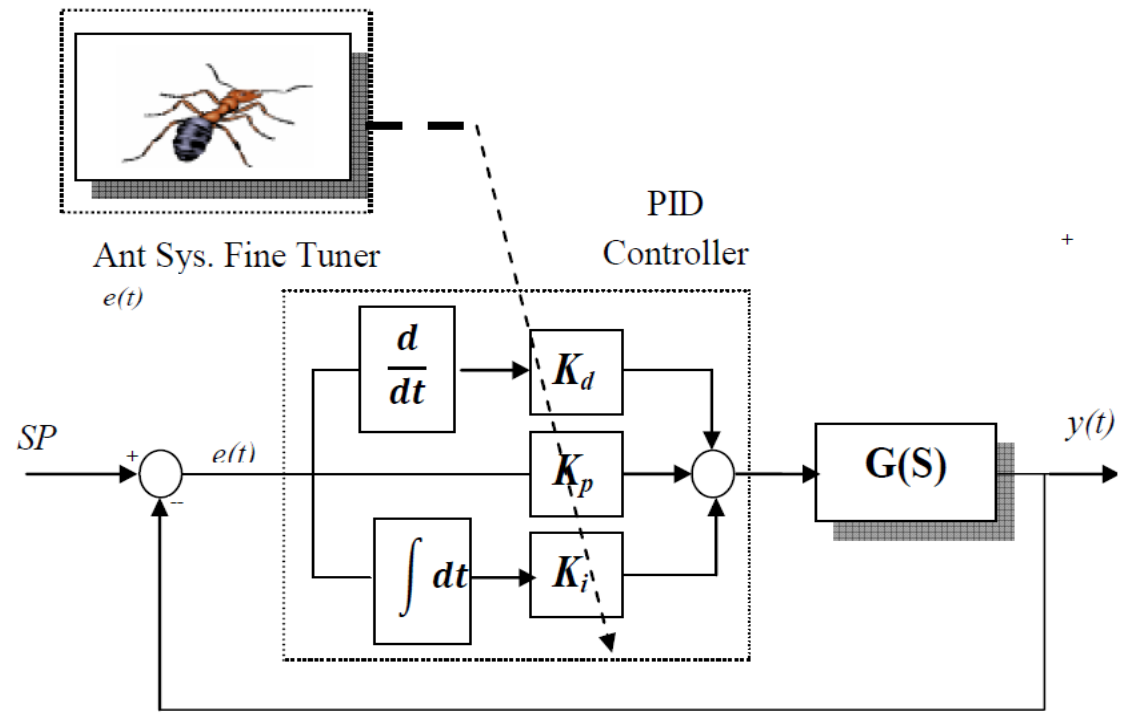

Fig (11) - The proposed closed loop control

Using ant system optimization frame work a planar structure of 10 rows and 15 lines as in Fig (12) is adopted. 10 rows mean the numbers of $0: 9$, and 15 lines mean 15 bits for the three gain parameters $\mathrm{Kp}, \mathrm{Ki}$ and $\mathrm{Kd}$. The nodes of lines 1, 2, 3, 4 and 5 are the 1st, 2nd, 3rd, 4th and 5th bits of Kp, lines 6, 7,8,9 and 10 are the 1st, 2nd, 3rd , 4th and 5th bits of Ki, lines $11,12,13,14$ and 15 are the 1st, 2nd , 3rd , 4th and 5th bits of Kd . So, in the ACS PID control, there are totally 150 nodes. nij is used to denote the node $\mathrm{j}$ on line $\mathrm{Li}$. The coordinate of the node nij is denoted by $\mathrm{j}$. Let an ant depart from the origin $\mathrm{O}$. In each step forward, it chooses a node from the next line $\mathrm{Li}(\mathrm{i}=1,2, \ldots, 15)$ and then moves to this node along the straight line. When it moves to a node on line L15, it completes one tour. Its moving path can be expressed as Path $=\{O, n 1 j, n 2 j, \ldots, n 15 j$.

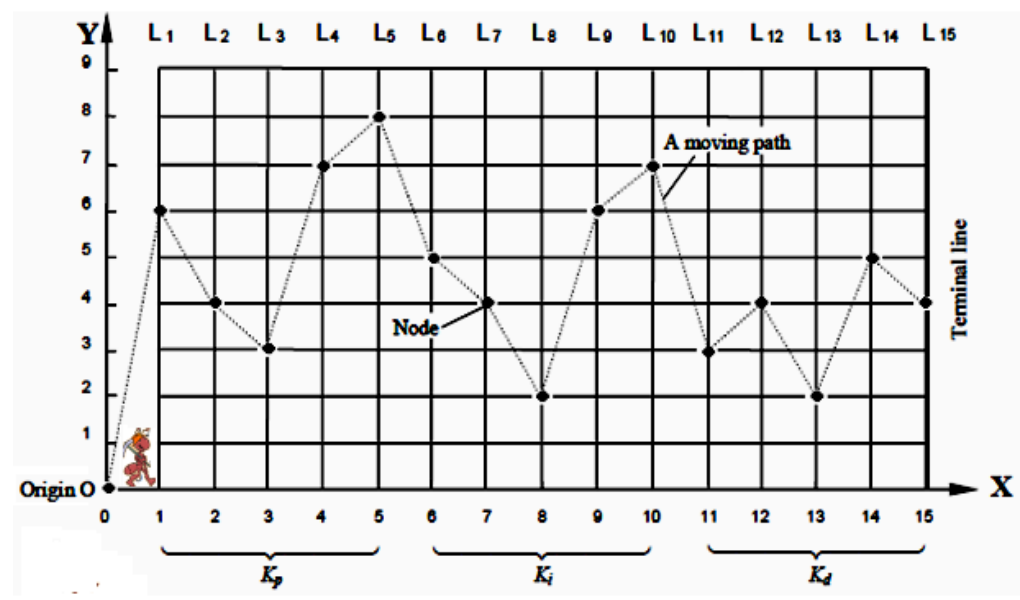

Fig (12) - Ant system optimization planar structures

The formulas give the values of $\mathrm{Kp}, \mathrm{K}_{\mathrm{i}}$ and $\mathrm{K}_{\mathrm{d}}$ represented by the paths and the steps of the ant colony algorithm are illustrated in detalies in [24-26]. 


\section{FUZZY SELF-TUNING OF PID DESIGN PROCEDURE}

The proposed design procedure includes two steps :

1. Find the optimal gains of PID based on the ACS.

2. Design fuzzy logic control (FLC) part, which has self-tuning capabilities for the obtained PID.

\section{Optimal PID controller}

The transfer function of the PID controller given in (5):

$$
\mathbf{k}(\mathbf{s})=\mathbf{k}_{\mathbf{p}}+\frac{\mathbf{k}_{\mathbf{i}}}{\mathbf{s}}+\mathbf{k}_{\mathbf{d}} \mathbf{s}
$$

where: $\mathrm{k}_{\mathrm{p}}, \mathrm{k}_{\mathrm{i}}$ and $\mathrm{k}_{\mathrm{d}}$ are the proportional, integral and differential gains respectively. The results of the optimal PID gains of the PMSM are tabulated in Table (2) :

Table (2) - PID Controller Gains

\begin{tabular}{|c|c|c|c|}
\hline & $\mathrm{k}_{\mathrm{p}}$ & $\mathrm{k}_{\mathrm{i}}$ & $\mathrm{k}_{\mathrm{d}}$ \\
\hline $\mathrm{ACS}$ & 60 & 20 & 3 \\
\hline
\end{tabular}

\section{On line Fuzzy Self Tuning for the PID Controller}

The proportional, integral and derivate $\left(\mathrm{K}_{\mathrm{P}}, \mathrm{K}_{\mathrm{I}}, \mathrm{K}_{\mathrm{D}}\right)$ gains in a system can be self-tuned on-line with the output of the system under control as shown in Fig (13). The designs steps of fuzzy self-tuning can be summarized as follows [27] :

1- Write the PID controller by the following equation :

$$
U=K_{p}+K_{I} \int e d t+K_{D} \frac{d e(t)}{d t}
$$

This equation of the PID after fuzzy effect can be written as :

where:

$$
U=K_{p 2}+K_{I 2} \int e d t+K_{D 2} \frac{d e(t)}{d t}
$$

$$
\begin{aligned}
& K_{p 2}=K_{p} * K_{P 1}, K_{I 2}=K_{I} * K_{I 1}, K_{D 2}=K_{D} * K_{D 1} \\
& \mathrm{~K}_{\mathrm{p} 1}, \mathrm{~K}_{\mathrm{I}}, \mathrm{K}_{\mathrm{D} 1} \text { are the gain outputs from fuzzy controller : }
\end{aligned}
$$

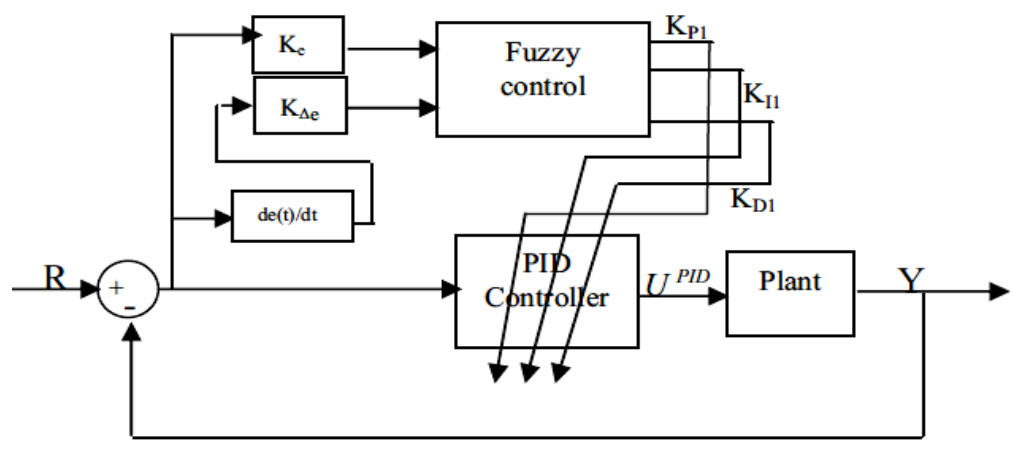

Fig (13) - Fuzzy self-tuned PID controller

2- Put the input membership functions of $\mathbf{e}$ and $\Delta \mathbf{e}$ as linguistic labels as shown in Fig (14). The inputs are \{Negative Big, Negative medium, Negative small, Zero, Positive small, 
Positive medium, Positive Big\}, and are referred to in the rules bases as $\{\mathrm{NB}, \mathrm{NM}, \mathrm{NS}, \mathrm{ZE}, \mathrm{PS}, \mathrm{PM}, \mathrm{PB}\}$ and the linguistic labels of the outputs are \{Zero, Medium small, Small, Medium, Big, Medium big, very big \} and referred to in the rules bases as $\{$ Z,MS, S, M, B, MB, VB\}. The output membership function is shown in Fig (15) [27].

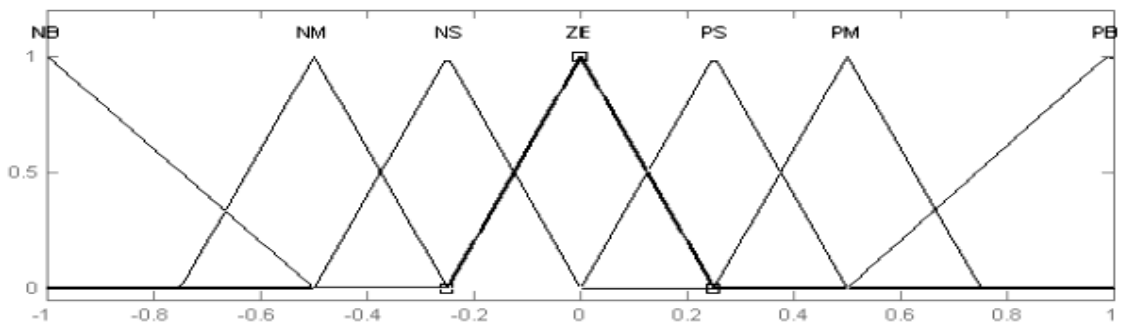

Fig (14) - Input membership functions of e and $\Delta e$

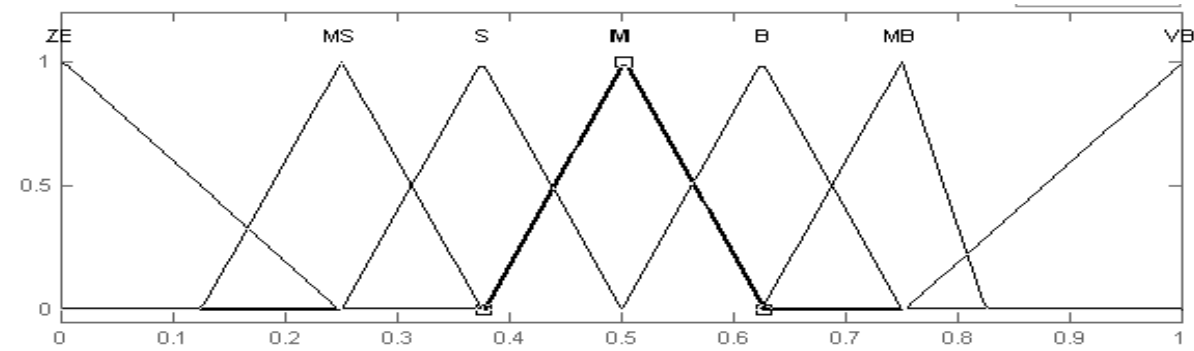

Fig (15) - Output membership functions

where:

Ke : error input normalizing gain.

$\mathrm{K} \Delta \mathrm{e}: \Delta$ error input normalizing gain.

3- Calculate the values of normalizing gain for e and $\Delta \mathrm{e}$. The Rule bases for determining $\mathrm{K}_{\mathrm{P} 1}, \mathrm{~K}_{\mathrm{I} 1}$ and $\mathrm{K}_{\mathrm{D} 1}$ are given in [27]. The defuzzification method used is the center of gravity. The detailed of this procedure is given and studied in [27].

VI. SIMULATION AND PRACTICAL RESULTS The laboratory consists in a Synchronous 3-phase servo motor permanent magnet with model SEW as shown in figures (1) and (2). The proposed controllers optimal PID and FSTOPID are coded using the MATLAB package and Simulink Toolbox based on multifunctional DAQ. The real-time simulation results for the PMSM and its drive system controlled are implemented as given in Fig (16). In order to clarify the improvements of the proposed controller, the simulation results with an optimal PID controller also are implemented for comparison purposes. The speed to volt and volt to speed transformation are given in Fig (17) and (18). In addition, the FSTOPID controller is shown in Fig (19). The system parameters of the 3-phase permanent magnet synchronous servo motor are given in the appendix.

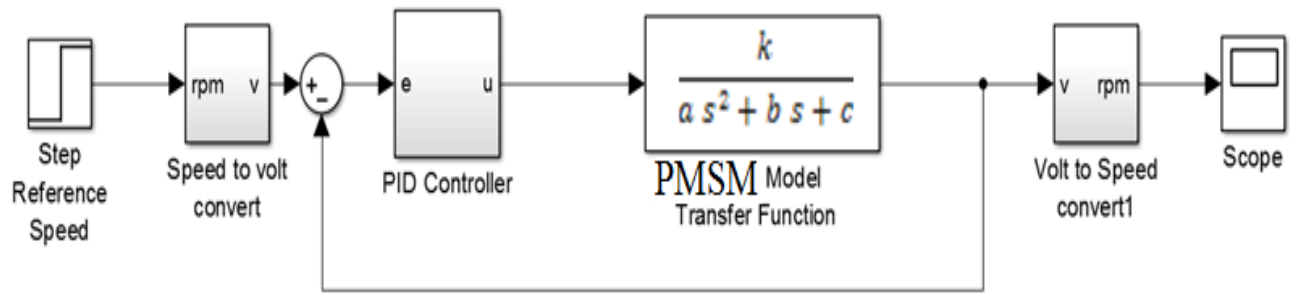

Fig (16) - PID controller for PMSM model. 


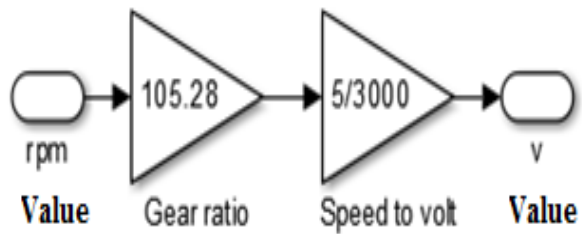

Fig (17) - Block of speed / volt converter

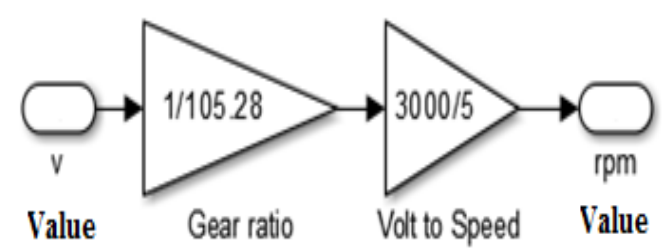

Fig (18) - Block of volt / speed converter

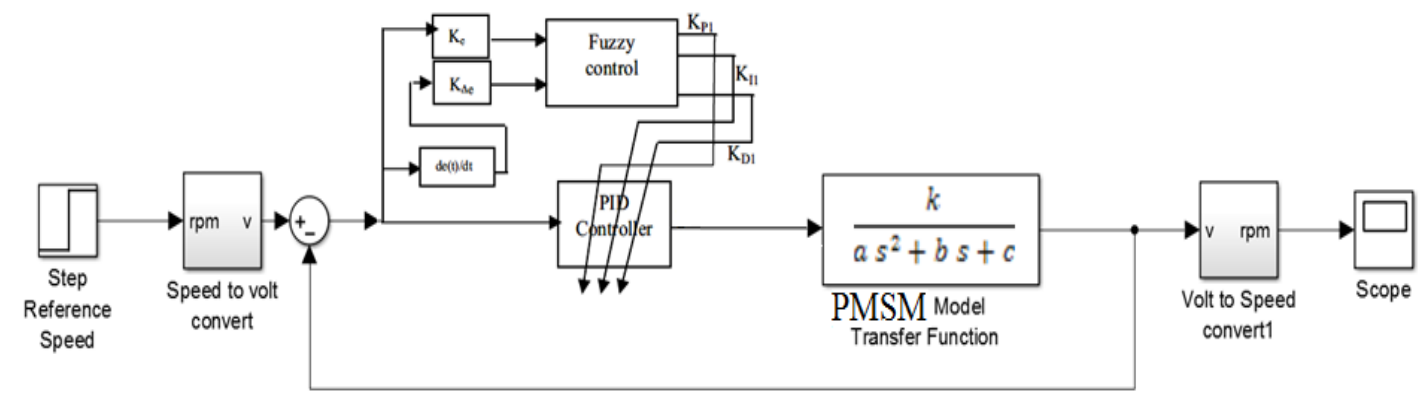

Fig (19) - Fuzzy Self Tune PID controller for PMSM Model.

The purpose of this part is to show the validity and real implantation of the proposed optimal PID and FSTOPID controllers as applied to a PMSM servo system. All controllers are applied to control the speed of the PMSM servomotor. The desired speed equivalent to 2 volt under normal load torque. Three tests of practical implementation are carried out.

\section{Test 1: Normal Load Torque and Step disturbance Speed (r.p.m) $=2 v$}

In this test, the effectiveness of the system equipped with the designed optimal ACS-PID compared with FSTOPID (real and simulation) is displayed in Fig (20). The corresponding outputs of the controllers are illustrated in Fig (21).

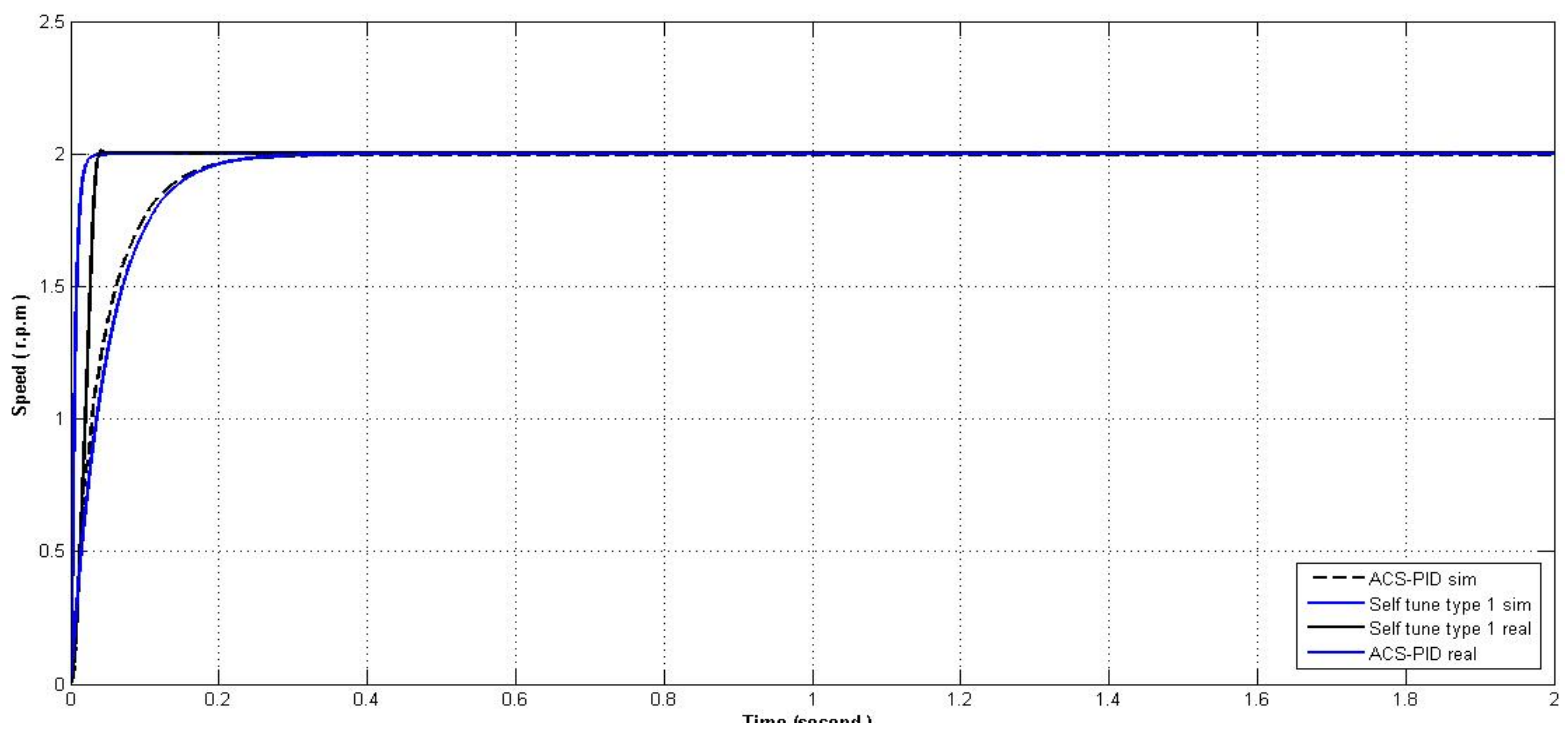

Fig (20) - PMSM system response with PID 


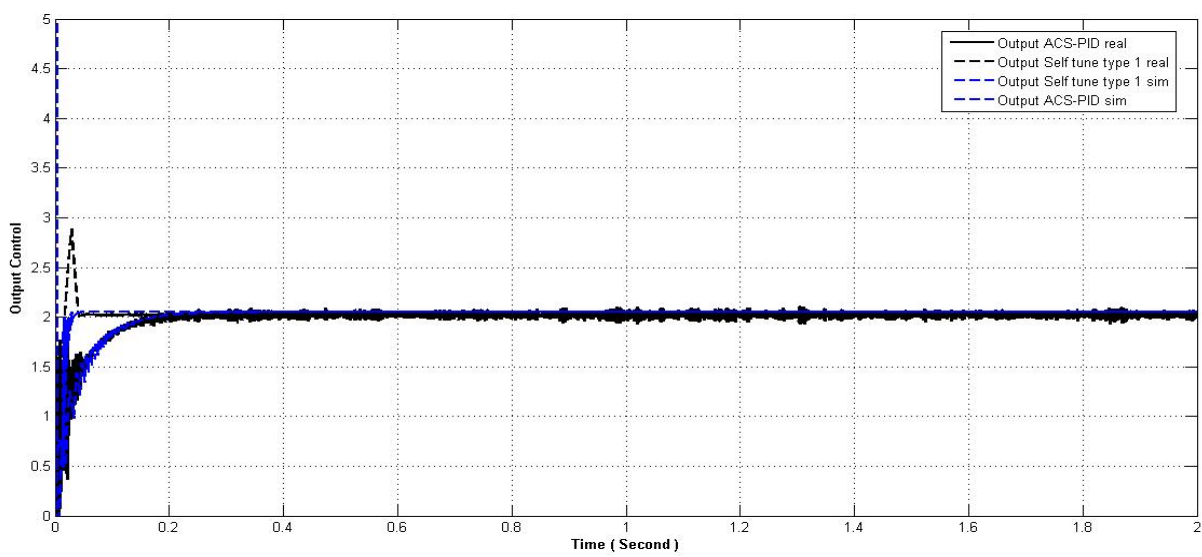

Fig (21) - Controller output for PID and fuzzy self-tuning of PID

From the previous two figures, it is worth mentioning that the real and simulation results are approximately close to each other. The FSTOPID decreases the settling time and removes the steady-state error faster than the PID. In addition the output of the controllers are within the practical PMSM range.

Test 2: Under Load Torque and Step disturbance Speed (r.p.m) $=2 v$

To test the effectiveness of the proposed controllers, suddenly increase change in the speed by $0.5 \underline{v}$ at time $(1.5 \mathrm{sec})$ is performed. This leads to decrease the load torque. From the speed time responses shown in Fig (22), a lower overshoot and smaller settling time are seen using FSTOPID than the optimal PID. The time response of the controller outputs are displayed in Fig (23. The controller output of FSTOPID for practical simulation has a small overshoot but it gives better response at this point for speed regulation.

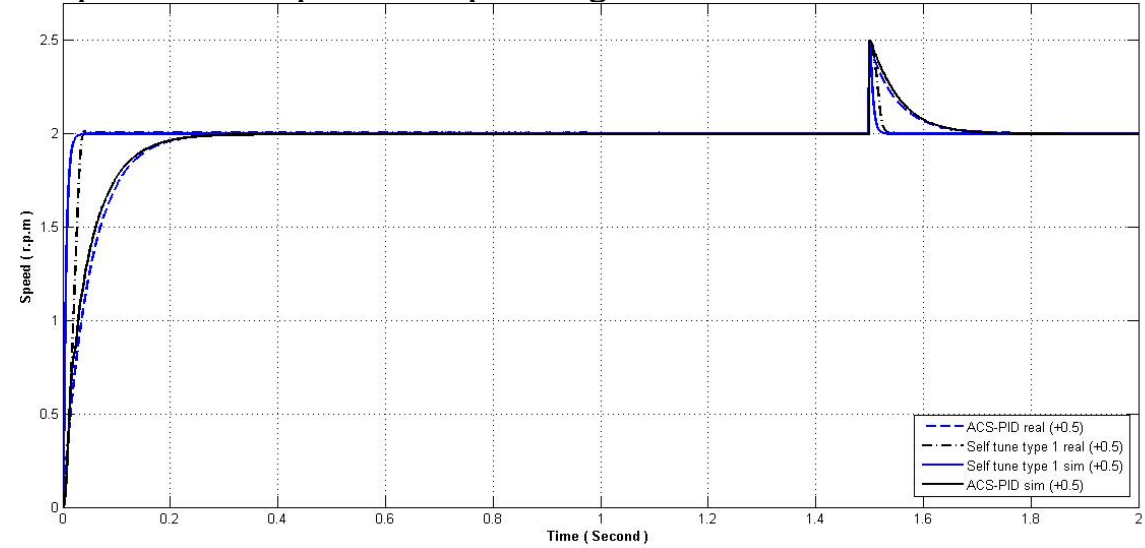

Fig (22)-. PMSM system response with PID

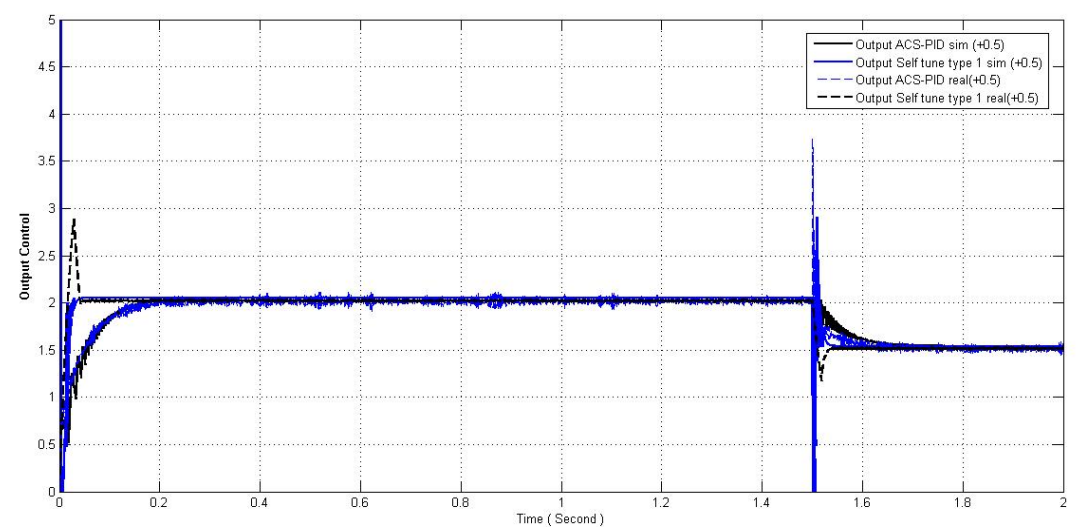

Fig (23) - Controller output for PID and fuzzy self-tuning of PID 
Test 3: Over Load Torque and Step disturbance Speed ( r.p.m) $=2 \mathrm{v}$

To test the effectiveness of the proposed controllers suddenly decrease in the speed by $0.5 \underline{v}$ at time $(1.5 \mathrm{sec})$ is performed. This cusses increasing on the load torque and consequentially the speed decreases. The proposed FSTOPID is found to produce better performance in the rise time, peak overshoot and the steady state error, settling time and delay time than optimal PID as depicted in Fig (24). The time response of the controller outputs are displayed in Fig (25). The controller output of FSTOPID for practical simulation has a overshoot but the value of the controller output steel with acceptable range and gives better response for corresponding speed regulation.

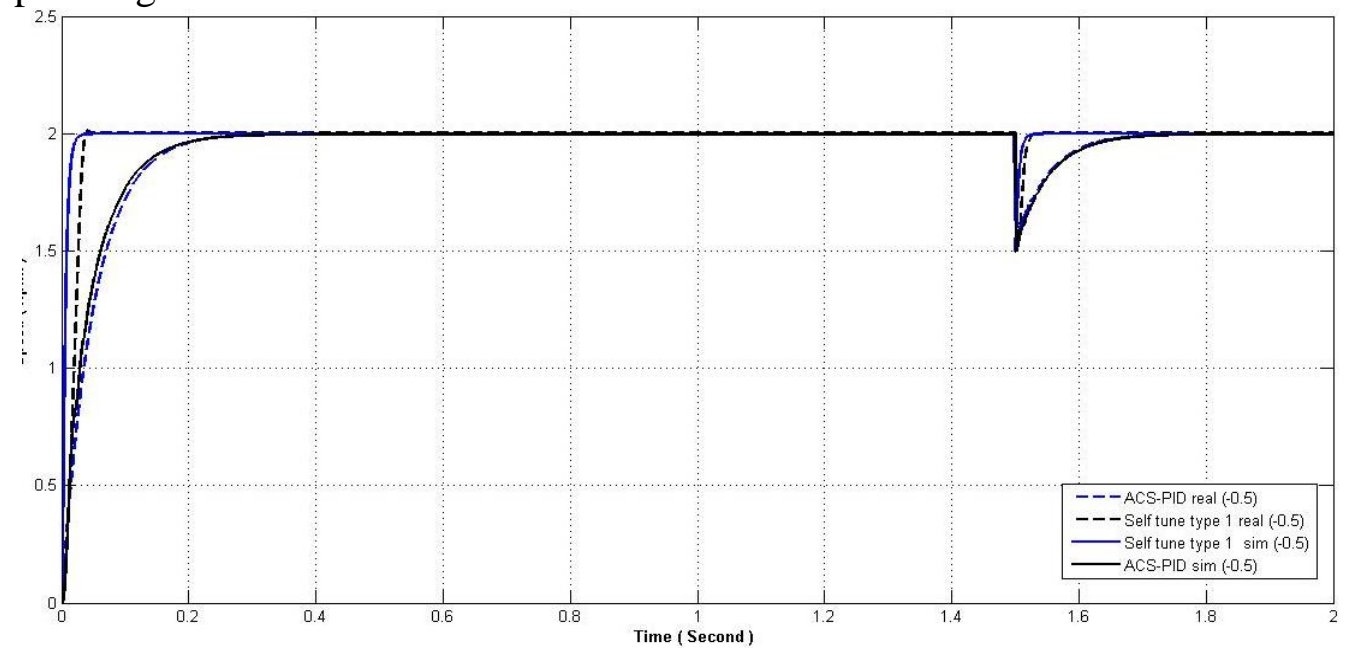

Fig (24) - PMSM system response with PID

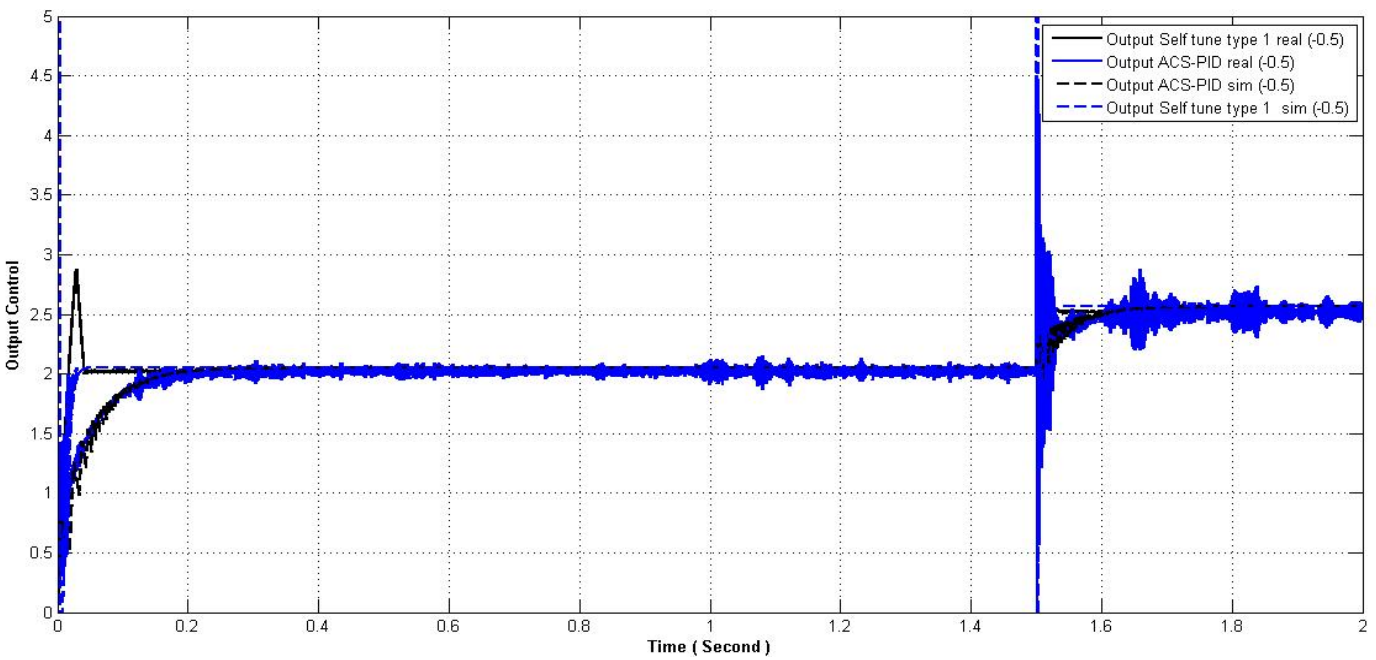

Fig (23) - Controller output for PID and fuzzy self-tuning of PID

\section{CONCLUSION}

Optimal PID and fuzzy logic system are combined to produce hybrid controller used to enhance the speed regulation of servo permanent magnet synchronous motor. According to the lack of the some driver parameters, nonlinear least-squares method is employed for the PMSM identification. The transient response of FSTOPID (for real and simulation tests) is faster to eliminate steady state error with minimum settling time than the optimal PID. The real and simulation results show approximately similar responses for optimal PID and FSTPID controllers. The proposed approach has superior features including easy implementation, good computational with keeping the system error closer to zero. 


\section{References}

[1] Boileau T, Leboeuf N, Nahid-Mobarakeh B., "Online identification of PMSM parameters: parameter identifiability and estimator comparative study", IEEE Transactions on Industrial Electronics 2011; 47(4) : 44 - 57.

[2] Luo Y, ChenY, Pi Y. Cogging, "Effect minimization in PMSM position servo system using dual high-order periodic adaptive learning compensation", ISA Transactions 2010 ;49(4) : $79-88$.

[3] Liu H, Li S., "Speed control for PMSM servo system using predictive functional control and extended state observer", IEEE Transactions on Industrial Electronics 2012; 59(2) :71-83.

[4] Zhang B, Pi Y, Luo Y., "Fractional order sliding-mode control based on parameters auto-tuning for velocity control of permanent magnet synchronous motor", ISA Transactions 2012; 51(5): $49-56$.

[5] Mohamed YA-RI., "Adaptive self-tuning speed control for PMSM with dead-time", IEEE Transactions on Energy Conversion 2006; 21(4) : 55 - 62.

[6] Sepe R, Lang J., "Real-time observer-based (adaptive) control of a permanent-magnet synchronous motor without mechanical sensors", IEEE Transactions on Industry Applications 1992; 28(6) : 45 - 52.

[7] Jan Rong-Maw, Tseng Chung-Shi, Liu Ren-Jun, "Robust PID control design for permanent magnet synchronous motor: a genetic approach", Electric Power Sys Res 2008;78:1161-8.

[8] Espina Jordi, Arias Antoni, Balcells Josep, Ortega Carlos, "Speed anti-windup PI strategies review for field oriented control of permanent magnet synchronous machines", IEEE Power Elect Contr Power Syst 2009 : 279 - 85.

[9] Lee Seok-Beom, "Closed-loop estimation of permanent magnet synchronous motor parameters by PI controller gain tuning”, IEEE Trans Energy Conv. 2006;21(4) : 63-70.

[10] Choi HH., "LMI-based nonlinear fuzzy observer-controller design for uncertain MIMO nonlinear systems", IEEE Trans Fuzzy Systems 2007; 15(5) : 56 - 71.

[11] Cheng M, Sun Q, Zhou E., "New self-tuning fuzzy PI control of a novel doubly salient permanent-magnet motor drive", IEEE Trans Ind Electronics 2006; 53(3) : 14-21.

[12] Feng G., "A survey on analysis and design of model-based fuzzy control systems", IEEE Trans Fuzzy Systems 2006; 14(6) : 76 - 97.

[13] Kung YS, Huang CC, Tsai MH., "FPGA realization of an adaptive fuzzy controller for PMLSM drive", IEEE Trans Ind. Electronics 2009; 56(8) : 2923 - 32.

[14 Li S, Liu Z., "Adaptive speed control for permanent-magnet synchronous motor system with variations of load inertia", IEEE Trans Ind. Electronics 2009; 56(8) : 3050 - 9.

[15] Cao Xianqing, Fan Liping, "Self-tuning PI controller for permanent magnet synchronous motor based on iterative learning control", IEEE second international symposium on intelligent information technology application; 2008. pp $756-60$.

[ 16] Wang Y, Shao H., "Optimal tuning for PI controller", Automatica 2000; 36 : 147 - 52.

[17] Jan Rong-Maw, Tseng Chung-Shi, Liu Ren-Jun, "Robust PID control design for permanent magnet synchronous motor: a genetic approach", Electric Power Sys. Res 2008; $78: 1161-8$.

[18] Espina Jordi, Arias Antoni, Balcells Josep, Ortega Carlos, "Speed anti-windup PI strategies review for field oriented control of permanent magnet synchronous machines", IEEE Power Electr Contr Power Syst 2009 : 279-85.

[19] Lee Seok-Beom, "Closed-loop estimation of permanent magnet synchronous motor parameters by PI controller gain tuning", IEEE Trans Energy Convers 2006; 21(4) : 863-70.

[20] D. Luczak and K. Zawirski, "Parametric Identification of Multi-Mass Mechanical Systems in Electrical Drives using Nonlinear Least Squares Method", IECON 2015kohama, PP40-46-40-51, November 9-12, 2015

[21] Kaiyu Wang, John Chiasson, Marc Bodson and Leon M. Tolbert, "A Nonlinear LeastSquares Approach for Identification of the Induction Motor Parameters", $43^{\text {rd }}$ IEEE Conference on Decision and Control December 14-17, Atlantis, Paradise Island, Bahamas pp 3856- 3861, 2004 
[22] M. V'elez-Reyes, K. Minami, and G. Verghese, "Recursive speed and parameter estimation for induction machines", Proceedings of the IEEE Industry Applications Conference, 1989. San Diego, California

[23] Q. Zeng and G. Tan, "Optimal Design of PID Controller Using Modified Ant Colony System Algorithm", IEEE, $3^{\text {rd }}$ International Conference on Natural Computation (ICNC), 2007

[24] M. Omar, M. Soliman, A. M. Abdel Ghany and F. Bendary, "Optimal Tuning of PID Controllers for Hydrothermal Load Frequency Control Using Ant Colony Optimization", Accepted to be published in IJEEI Journal , October 2013.

[25] M. Omar, M. Soliman, A. M. Abdel Ghany and F. Bendary, "Ant Colony Optimization based PID for single Area Load Frequency Control", Accepted to be published in ICMIC Conference September 2013.

[26] M. A. Abdel Ghany, M. E. Bahgat, W. M. Refaey and F. N. Hassan, "Ant Colony Optimum Tuning of PID Load Frequency Controller for the Egyptian Power System", Sixteenth International Middle East Power Systems Conference (MEPCON'14), Ain Shams University, Egypt, 2014.

[27] Maher M.F. Algreer and Yhya R.M.Kuraz, "Design Fuzzy Self Tuning of PID Controller for Chopper-Fed DC Motor Drive", Al-Rafidain Engineering Journal, Vol. 16, No. 2, pp. 54-66 A, 2008.

\section{Appendix}

The 3-phase synchronous servo motor parameters are illustrated as follows :

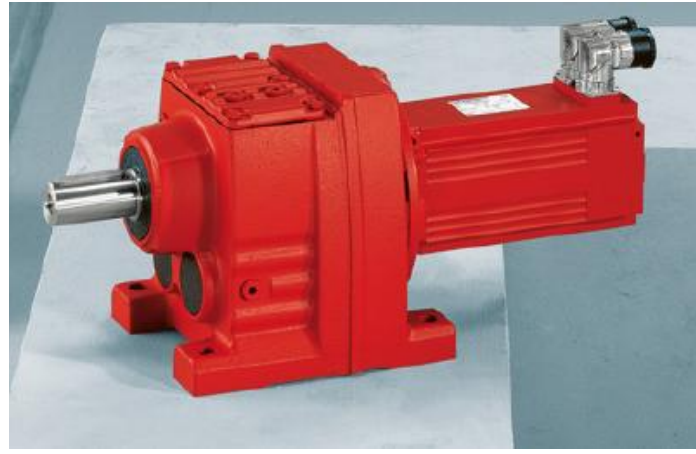

Fig (A) - 3-phase synchronous servo motor

Table (3) - The parameters of PMSM

Parameters

\begin{tabular}{|c|c|c|}
\hline$K_{t}(N m / A)$ & 0.61 & $\begin{array}{c}\text { Torque constant } \\
=M_{0}(\text { standstill torque }) / I_{0}(\text { standstill current }) \\
=1 \mathrm{Nm} / 1.65 \mathrm{~A}\end{array}$ \\
\hline $\boldsymbol{R}_{a}(\boldsymbol{\Omega})$ & 5.7 & Resistance between connection phase and star point \\
\hline 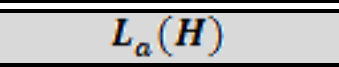 & 0.0097 & Inductivity between connection phase and star point \\
\hline Tm(my.me & 0.0010 & 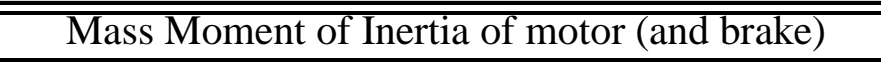 \\
\hline$B_{m}(\mathrm{~N} . \mathrm{m} / \mathrm{rad} / \mathrm{s})$ & $3.5 \times 10^{-4}$ & Viscous Damping on the motor shaft \\
\hline$I_{g}\left(\mathrm{~kg} \cdot \mathrm{m}^{2}\right)$ & $0.12 \times 10^{-4}$ & $\begin{array}{c}\text { Mass Moment of Inertia of gear unit referred to the } \\
\text { motor shaft }\end{array}$ \\
\hline$B_{g}(N . m / r a d / s)$ & 509 & Viscous Damping on the gear unit output shaft \\
\hline $\bar{N}$ & 105.28 & Gear Unit Reduction Ratio \\
\hline
\end{tabular}

